\title{
Mutillidae (Hymenoptera, Aculeata) do estado de Mato Grosso do Sul, Brasil
}

\author{
David Richard Luz' ${ }^{1}$ Rodrigo Aranda ${ }^{2} \&$ Kevin Andrew Williams ${ }^{1}$
}

\begin{abstract}
1. Laboratório de Biologia Comparada de Hymenoptera, Departamento de Zoologia, Universidade Federal do Paraná, Caixa Postal 19020, 81531-980, Curitiba, PR, Brasil. (drdaluz@gmail.com)

2. Programa de Pós-Graduação em Ecologia e Conservação, Centro de Ciências Biológicas e da Saúde, Cidade Universitária s/n Caixa Postal 549, 79070-900, Campo Grande, MS, Brasil.
\end{abstract}

Recebido 21 novembro 2016

Aceito 6 fevereiro 2017

DOI: $10.1590 / 1678-4766 e 2017124$

\begin{abstract}
Mutillidae (Hymenoptera, Aculeata) of the state of Mato Grosso do Sul, Brazil. Mutillid wasps are parasitoids of mature larvae or pre-pupae of other insects, especially other aculeate Hymenoptera. They are characterized by a strong sexual dimorphism: all females are wingless, while males of most species have wings. The family comprises about 4,200 species and their greatest diversity occurs in the tropical and subtropical regions of the world. Despite the size of the family in the Neotropical Region (about 1,500 species, 560 in Brazil), surveys of the Mutillidae fauna are scarce. The purpose of this work is to present an updated list of Mutillidae species recorded for the state of Mato Grosso do Sul, Brazil, based on literature, museum records and new data. A total of 114 species (including 22 morphospecies) distributed in 23 genera, three tribes and two subfamilies were recorded. The richest genera were Traumatomutilla André, 1901 (36 species), Timulla Ashmead, 1899 (14 spp.), Ephuta Say, 1836 (11 spp.) and Hoplomutilla Ashmead, 1899 (10 spp.).
\end{abstract}

KEYWORDS. Solitary wasp, Neotropical, Biota-MS Program.

RESUMO. Mutilídeos são parasitóides de formas imaturas de outros insetos, especialmente Hymenoptera aculeados. Eles são caracterizados pelo seu acentuado dimorfismo sexual: todas as fêmeas são ápteras, enquanto a maioria dos machos é alada. A família compreende cerca de 4.200 espécies e sua maior diversidade é encontrada nas regiões tropicais e subtropicais do mundo. Apesar do tamanho da família na Região Neotropical (cerca de 1.500 espécies, sendo 560 no Brasil), levantamentos da fauna de Mutillidae são escassos. O objetivo deste trabalho é apresentar uma lista atualizada das espécies de Mutillidae presentes no estado de Mato Grosso do Sul, Brasil, incluindo registros retirados da literatura, de coleções científicas e de coletas realizadas recentemente no estado. Um total de 114 espécies (incluindo 22 morfoespécies), distribuídas em 23 gêneros, três tribos e duas subfamílias foram registradas. Os gêneros com o maior riqueza foram Traumatomutilla André, 1901 (36 espécies), Timulla Ashmead, 1899 (14 spp.), Ephuta Say, 1836 (11 spp.) e Hoplomutilla Ashmead, 1899 (10 spp.).

PALAVRAS-CHAVE. Vespas solitárias, Neotropical, Programa Biota-MS.

A família Mutillidae compreende um grupo monofilético (BRothers, 1975; BROTHERs \& CARPENTER, 1993) de vespas solitárias aculeadas (Hymenoptera, Aculeata) que apresentam acentuado dimorfismo sexual e são parasitoides de imaturos de outros insetos, especialmente abelhas e vespas apoideas (Brothers, 1989). A família é cosmopolita e a maior diversidade ocorre nas regiões tropicais e subtropicais do mundo (BRothers, 1975). Atualmente são reconhecidas cerca de 4.200 espécies (LELEJ, 2007) distribuídas em 208 gêneros (LELEJ \& BROTHERS, 2008). Das sete subfamílias reconhecidas para a família (sensu Brothers, 1975, 1999) apenas duas, Mutillinae e Sphaeropthalminae, ocorrem na Região Neotropical, com aproximadamente 1.500 espécies (NoNVEILleR, 1990).

Diferentemente do que acontece em outros grupos de insetos, inventariamentos da fauna de Mutillidae de uma determinada localidade ou país são extremamente raros na
Região Neotropical. Na Guiana, Mickel (1952), ao estudar material depositado em várias coleções, indicou 65 espécies. No Panamá, após nove extensivos anos de coletas em um grande número de localidades, CAMBRA \& QuintERo (1992) encontraram 67 espécies e CAMBRA (1997), ao abordar apenas a subfamília Sphaeropthalminae, registrou 65 espécies. No Paraguai, Quintero \& CAMBra (1996a) estudaram o material depositado no Museo Nacional de Historia Natural del Paraguay e reportaram 134 espécies. No Peru, QuINTERO \& CAMBRA (1996b) realizaram três viagens de coleta entre os anos de 1990 e 1992 e encontraram 133 espécies. Na Costa Rica, ao estudar apenas Sphaeropthalminae, CAMBRA (1997) registrou 79 espécies. Na Argentina, FrITZ (2008) indicou a presença de 317 espécies. Em El Salvador, CAMBRA \& Sermeño (2001) estudaram os espécimes do Museo de Invertebrados G. B. Fairchild, Universidad de Panamá e na Universidad de El Salvador e encontraram 42 espécies. 
No Brasil, GARCIA (2004) realizou coletas sistematizadas entre os anos de 2002 e 2003 no município de Piraquara, Paraná e registrou 27 espécies. No estado de Mato Grosso do Sul, Aranda \& Catian (2008), entre os anos de 2005 e 2007 , conduziram coletas sistematizadas em sete localidades distintas da Serra da Bodoquena e verificaram 23 espécies. A fim de aumentar o conhecimento da fauna de Mutillidae de Mato Grosso do Sul, objetiva-se apresentar uma lista atualizada das espécies registradas para o estado.

\section{MATERIAL E MÉTODOS}

A lista de espécies foi produzida a partir de dados obtidos através de três fontes de informação:

Coletas em campo. Entre os anos de 2005 e 2013, exemplares de Mutillidae foram coletados em seis diferentes localidades do estado de Mato Grosso do Sul: Parque Nacional da Serra da Bodoquena ( $\left.21^{\circ} 00^{\prime} \mathrm{S}, 56^{\circ} 41^{\prime} \mathrm{W}\right)$, Base de Estudo do Pantanal - UFMS (19 $\left.19^{\circ} 35^{\prime} \mathrm{S}, 56^{\circ} 57^{\prime} \mathrm{W}\right)$, Fazenda Nhumirim Embrapa Pantanal (18 $\left.59^{\circ} \mathrm{S}, 56^{\circ} 39^{\prime} \mathrm{W}\right)$, Dourados ( $22^{\circ} 13^{\prime} \mathrm{S}$, $\left.54^{\circ} 48^{\prime} \mathrm{W}\right)$, Três Lagoas $\left(20^{\circ} 45^{\prime} \mathrm{S}, 52^{\circ} 31^{\prime} \mathrm{W}\right)$ e em fragmentos de cerrado de Campo Grande (20 $\left.28^{\circ} \mathrm{S}, 54^{\circ} 32^{\prime} \mathrm{W}\right)$. O material coletado está depositado no Museu de Biodiversidade da Universidade Federal da Grande Dourados (Mubio-UFGD) e na Coleção Zoológica da Universidade Federal de Mato Grosso do Sul (ZUFMS);

Consulta ao acervo de coleções. Os exemplares provenientes do Mato Grosso do Sul e depositados nas coleções do Museu de Zoologia da Universidade de São Paulo (MZSP), do Museu Nacional, Universidade Federal do Rio de Janeiro (MNRJ), do Museu de Entomologia da FEIS/Unesp (MEFEIS) e da Coleção Entomológica Pe. Jesus Santiago Moure, Departamento de Zoologia, Universidade Federal do Paraná (DZUP) foram catalogados;

Consulta à literatura histórica. A extensa literatura contemplando os mutilídeos do Brasil foi integralmente examinada e todas as espécies reportadas para o estado de Mato Grosso do Sul, tanto em trabalhos de descrição de espécies como revisão de gêneros, foram também analisados.

\section{RESULTADOS E DISCUSSÃO}

São registradas 114 espécies (incluindo 22 morfoespécies) de Mutillidae para as diferentes localidades do estado de Mato Grosso do Sul (Fig. 1, Tab. I). Os táxons estão distribuídas em 23 gêneros, três tribos e duas subfamílias. Além dessas espécies, são reportados na literatura dois nomes incertae sedis (NONVEILLER, 1990) descritos para o município de Corumbá por E. T. Cresson: Mutilla tantula Cresson, 1902, fêmea, e Mutilla turnalis Cresson, 1902, fêmea.

Os gêneros com o maior número de espécies registradas foram Traumatomutilla André, 1901 (36 espécies e duas subespécies), Timulla Ashmead, 1899 (14 spp.), Ephuta Say, 1836 (11 spp.) e Hoplomutilla Ashmead, 1899 (10 spp.). De acordo com NonveILLER (1990), esses quatro

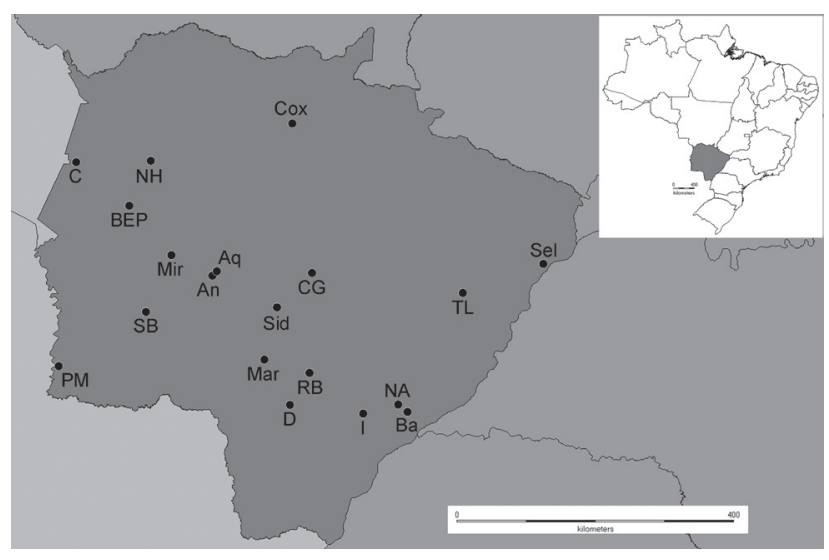

Fig. 1. Mapa com as localidades de coleta das espécies de Mutillidae registradas para o estado de Mato Grosso do Sul neste estudo (Na, Anastácio; Aq, Aquidauana; Ba, Batayporã; BEP, Base de Estudo do Pantanal (UFMS); C, Corumbá; CG, Campo Grande; Cox, Coxim; D, Dourados; I, Ivinhema; Ma, Maracaju; Mir, Miranda; NA, Nova Andradina; NH, Fazenda Nhumirim (Embrapa Pantanal); PM, Porto Murtinho; RB, Rio Brilhante; SB, Serra da Bodoquena; Sel, Selvíria; Sid, Sidrolândia; TL, Três Lagoas).

gêneros são aqueles que apresentam a maior riqueza de espécies na Região Neotropical, representando juntos cerca de $44 \%$ de todas as espécies conhecidas.

Por outro lado, gêneros que possuem conhecida alta diversidade foram pouco representados neste estudo. Pseudomethoca Ashmead, 1896, amplamente distribuído na América do Sul (Quintero \& CAMBra, 1996b), teve quatro espécies registradas. Darditilla Casal, 1965, gênero que possui a sua maior diversidade na porção sul do continente sulamericano (CASAL, 1968; BROTHERS, 2006), foi representado por apenas cinco espécies. Situação interessante ocorre em Ephuta, gênero com o maior número de espécies na Região Neotropical (Nonveiller, 1990; Quintero \& Cambra, 1996b): apesar de ser listado como um dos gêneros mais ricos no presente estudo (11 spp.), a diversidade total deste gênero no Mato Grosso no Sul é certamente muito maior do que aquela aqui apresentada. Comparativamente, GARCIA (2004), ao realizar coletas sistemáticas em uma área restrita no município de Piraquara, Paraná, encontrou seis espécies de Pseudomethoca, três de Darditilla e nove de Ephuta. No Paraguai, Quintero \& CAMBra (1996a) registraram 12 espécies de Pseudomethoca, nove de Darditilla e 14 de Ephuta.

Como pode ser observado na Tabela I, apenas Timulla (Timulla) terminalis (Gerstaecker) é conhecida para os dois sexos. A grande maioria das espécies aqui apresentadas (96) é conhecida unicamente para as fêmeas, enquanto outras 17 apenas para machos. Segundo Pilgrim \& PitTs (2006), considerando apenas os mutilídeos diurnos do Novo Mundo, $64 \%$ das espécies foram descritas utilizando unicamente fêmeas, $24 \%$ somente machos e apenas $12 \%$ das espécies descritas possuem os dois sexos associados.

Embora perturbador, esse cenário é comum dentro de Mutillidae, uma vez que a característica mais marcante é, sem dúvida, o seu acentuado dimorfismo sexual: todas as fêmeas 
Tab. I. Lista de espécies de Mutillidae registradas para o estado de Mato Grosso do Sul, Brasil, indicando: sexo conhecido (F, fêmeas; M, machos), Localidades (Na, Anastácio; Aq, Aquidauana; Ba, Batayporã; BEP, Base de Estudo do Pantanal (UFMS); C, Corumbá; CG, Campo Grande; Cox, Coxim; D, Dourados; I, Ivinhema; Ma, Maracaju; Mir, Miranda; NA, Nova Andradina; NH, Fazenda Nhumirim (Embrapa Pantanal); PM, Porto Murtinho; RB, Rio Brilhante; SB, Serra da Bodoquena; Sel, Selvíria; Sid, Sidrolândia; TL, Três Lagoas; ?, localidade desconhecida) e referência, se o registro foi retirado da literatura. ", novos registros para o estado; ${ }^{* *}$,novos registros para o Brasil.

\begin{tabular}{|c|c|c|c|}
\hline Subfamília/Tribo/Espécie & Sexo & Localidades & Referência \\
\hline \multicolumn{4}{|l|}{ SPHAEROPTHALMINAE } \\
\hline \multicolumn{4}{|l|}{ Sphaeropthalmini } \\
\hline Atillum albipictum Mickel, 1943 & $\mathrm{~F}$ & $\mathrm{TL}$ & \\
\hline Atillum feroculum Mickel, 1943 & $\mathrm{~F}$ & $\mathrm{C}, \mathrm{CG}$ & \\
\hline Atillum magisterium Mickel, 1943 & $\mathrm{~F}$ & C, Sid & Mickel, 1943 \\
\hline Calomutilla aff. crucigera (Burmeister, 1854)* & $\mathrm{F}$ & CG & \\
\hline Calomutilla sp. 1 & $\mathrm{~F}$ & SB & \\
\hline Cephalomutilla distincta Mickel, $1960^{* *}$ & $\mathrm{~F}$ & $\mathrm{NH}$ & \\
\hline Cephalomutilla transversa Mickel, 1960 & $\mathrm{~F}$ & PM & MiCKEL, 1960 \\
\hline Cephalomutilla vivata (Cresson, 1902) & $\mathrm{F}$ & $\mathrm{NH}, \mathrm{SB}, \mathrm{TL}$ & \\
\hline Darditilla araxa (Cresson, 1902) & $\mathrm{F}$ & $\mathrm{C}, \mathrm{CG}, \mathrm{TL}$ & Cresson, 1902 \\
\hline Darditilla debilis (Gerstaecker, 1874) & $\mathrm{F}$ & $\mathrm{SB}, \mathrm{TL}$ & \\
\hline Darditilla mita Casal, $1968^{* *}$ & $\mathrm{~F}$ & $\mathrm{C}$ & \\
\hline Darditilla vianai Casal, $1968^{* *}$ & $\mathrm{~F}$ & $\mathrm{TL}$ & \\
\hline Darditilla sp. 1 & $\mathrm{~F}$ & CG & \\
\hline Hoplocrates decumata Mickel, $1941^{* *}$ & M & CG & \\
\hline Hoplocrates monacha monacha (Gerstaecker, 1874) & $\mathrm{F}$ & $\mathrm{CG}, \mathrm{D}, \mathrm{SB}$ & \\
\hline Hoplocrates smithii (Cresson, 1902) & $\mathrm{F}$ & $\mathrm{TL}$ & \\
\hline Hoplomutilla alagoa (Cresson, 1902)* & M & CG & \\
\hline Hoplomutilla anthracina (Gerstaecker, 1874)* & M & CG & \\
\hline Hoplomutilla coimbra (Cresson, 1902) & M & $\mathrm{C}$ & Cresson, 1902 \\
\hline Hoplomutilla gigantea (Perty, 1833) & $\mathrm{F}$ & $\mathrm{C}$ & Cresson, 1902 \\
\hline Hoplomutilla goyazana (André, 1898) & $\mathrm{F}$ & $\mathrm{TL}$ & \\
\hline Hoplomutilla myops flaviomyops Mickel, 1939 & $\mathrm{~F}$ & $\mathrm{CG}, \mathrm{SB}, \mathrm{TL}$ & \\
\hline Hoplomutilla pollens (Kohl, 1882)* & $\mathrm{F}$ & Sel & \\
\hline Hoplomutilla sciron Mickel, $1939^{*}$ & $\mathrm{~F}$ & $\mathrm{NH}$ & \\
\hline Hoplomutilla serena (Gerstaecker, 1874$)^{*}$ & $\mathrm{~F}$ & NA & \\
\hline Hoplomutilla triumphans Mickel, 1939 & $\mathrm{~F}$ & C, PM, Sel, SB & MiCKeL, 1939 \\
\hline Horcomutilla fronticornis (Burmeister, 1854)* & $\mathrm{F}$ & TL & \\
\hline Horcomutilla sp. 1 & $\mathrm{~F}$ & $\mathrm{SB}$ & \\
\hline Leucospilomutilla staurogastra Suárez, 1973 & $\mathrm{~F}$ & Cox, TL & SuÁREZ, 1973 \\
\hline Lophomutilla obscura Fritz \& Pagliano, 1993 & $\mathrm{~F}$ & CG & \\
\hline Lophostigma sp. 1 & $\mathrm{~F}$ & CG & \\
\hline Lynchiatilla silvai Casal, $1963^{*}$ & $\mathrm{~F}$ & $\mathrm{CG}, \mathrm{SB}$ & \\
\hline Mickelia cressoni Suárez, 1966 & $\mathrm{~F}$ & Ma, Mir & SuÁREZ, 1966 \\
\hline Mickelia sp. 1 & $\mathrm{~F}$ & SB & \\
\hline Pertyella camposseabrai Casal, $1964^{*}$ & $\mathrm{~F}$ & Sel, NH & \\
\hline Pertyella aff. paulista Casal, 1964* & $\mathrm{F}$ & NH & \\
\hline Pertyella sp. 1 & $\mathrm{~F}$ & SB & \\
\hline Protophotopsis (Protophotopsiella) sulcifrons (André, 1906)* & $\mathrm{F}$ & Sel & \\
\hline Pseudomethoca gounellei (André, 1895)* & $\mathrm{F}$ & $\mathrm{TL}$ & \\
\hline Pseudomethoca sp. 1 & F & CG & \\
\hline Pseudomethoca sp. 2 & $\mathrm{~F}$ & $\mathrm{Aq}$ & \\
\hline Pseudomethoca sp. 3 & $\mathrm{~F}$ & SB & \\
\hline Ptilomutilla pennata André, 1905 & $\mathrm{~F}$ & SB & \\
\hline Sphaeropthalma sp. 1 & $\mathrm{~F}$ & $\mathrm{Aq}$ & \\
\hline Sphaeropthalma sp. 2 & M & $\mathrm{TL}$ & \\
\hline Suareztilla centrolineata (André, 1906) & $\mathrm{F}$ & $\mathrm{SB}, \mathrm{Sid}, \mathrm{TL}$ & MiCKeL, 1964 \\
\hline Suareztilla colorata (Gerstaecker, 1874) & M & $\mathrm{Ba}, \mathrm{C}$ & MiCKeL, 1964 \\
\hline Tallium maraye Casal, $1962^{* *}$ & $\mathrm{~F}$ & $\mathrm{RB}, \mathrm{TL}$ & \\
\hline Tallium precarium Suárez, 1960 & M & Ma & SUÁREZ, 1960 \\
\hline Tallium pretiosum (Gerstaecker, 1874$)^{* *}$ & $\mathrm{~F}$ & BEP & \\
\hline Tallium schusteri Suárez, 1960 & $\mathrm{~F}$ & $\mathrm{Ma}, \mathrm{RB}, \mathrm{TL}$ & SUÁREZ, 1960 \\
\hline Traumatomutilla barra (Cresson, 1902)* & M & $\mathrm{Ba}$ & \\
\hline Traumatomutilla bivittata bivittata (Gerstaecker, 1874)* & $\mathrm{F}$ & $\mathrm{CG}, \mathrm{SB}, \mathrm{TL}$ & \\
\hline Traumatomutilla bivittata rubroguttata André, $1901^{* *}$ & $\mathrm{~F}$ & An & \\
\hline Traumatomutilla caipira Casal, $1969^{*}$ & $\mathrm{~F}$ & $\mathrm{TL}$ & \\
\hline Traumatomutilla chilca Casal, $1969^{* *}$ & $\mathrm{~F}$ & $\mathrm{TL}$ & \\
\hline Traumatomutilla compar (André, 1895) & $\mathrm{F}$ & $\mathrm{C}$ & Cresson, 1902 \\
\hline Traumatomutilla contempta André, $1908^{* *}$ & $\mathrm{~F}$ & $?$ & \\
\hline Traumatomutilla crixa (Cresson, 1902)* & $\mathrm{F}$ & TL & \\
\hline Traumatomutilla demissa (Cresson, 1902)* & $\mathrm{F}$ & $\mathrm{TL}$ & \\
\hline
\end{tabular}


Tab. I. Cont.

\begin{tabular}{|c|c|c|c|}
\hline Subfamília/Tribo/Espécie & Sexo & Localidades & Referência \\
\hline Traumatomutilla dubia albata (Smith, 1879) & F & TL & \\
\hline Traumatomutilla estrella (Cresson, 1902) & $\mathrm{F}$ & $\mathrm{C}$ & Cresson, 1902 \\
\hline Traumatomutilla gausapata Mickel, $1952^{*}$ & $\mathrm{~F}$ & $\mathrm{TL}$ & \\
\hline Traumatomutilla geographica (Gerstaecker, 1874) & $\mathrm{F}$ & $\mathrm{TL}$ & \\
\hline Traumatomutilla gemina (Gerstaecker, 1874$)^{*}$ & $\mathrm{~F}$ & $?$ & \\
\hline Traumatomutilla graphica (Gerstaecker, 1874) & $\mathrm{F}$ & An, Aq, C, SB & Cresson, 1902 \\
\hline Traumatomutilla gurisa Casal, $1969^{* *}$ & $\mathrm{~F}$ & TL & \\
\hline Traumatomutilla indicoides Mickel, $1945^{* *}$ & $\mathrm{~F}$ & Sel, TL & \\
\hline Traumatomutilla inermis inermis (Klug, 1821) & F & SB & \\
\hline Traumatomutilla juvenilis (Gerstaecker, 1874)* & $\mathrm{F}$ & $\mathrm{NH}, \mathrm{TL}$ & \\
\hline Traumatomutilla latevittata (Cresson, 1902)* & $\mathrm{F}$ & $\mathrm{TL}$ & \\
\hline Traumatomutilla lineifera André, 1903* & $\mathrm{F}$ & NA & \\
\hline Traumatomutilla manca (Cresson, 1902) & $\mathrm{F}$ & $\mathrm{SB}, \mathrm{TL}$ & \\
\hline Traumatomutilla maula Casal, $1969^{*}$ & $\mathrm{~F}$ & TL & \\
\hline Traumatomutilla moesta (Gerstaecker, 1874)* & $\mathrm{F}$ & $\mathrm{TL}$ & \\
\hline Traumatomutilla obsoleta (Klug, 1821)* & $\mathrm{F}$ & $\mathrm{TL}$ & \\
\hline Traumatomutilla ocellaris (Klug, 1821) & $\mathrm{F}$ & $\mathrm{TL}$ & \\
\hline Traumatomutilla parallela (Klug, 1821) & $\mathrm{F}$ & $\mathrm{C}, \mathrm{TL}$ & Cresson, 1902 \\
\hline Traumatomutilla peperina Casal, $1969^{* * *}$ & $\mathrm{~F}$ & $\mathrm{TL}$ & \\
\hline Traumatomutilla quadrinotata (Klug, 1821)* & $\mathrm{F}$ & $\mathrm{C}, \mathrm{D}$ & \\
\hline Traumatomutilla quadrum (Klug, 1821)* & $\mathrm{F}$ & $\mathrm{CG}, \mathrm{TL}$ & \\
\hline Traumatomutilla serra (Cresson, 1902)* & M & NA, TL & \\
\hline Traumatomutilla solemnis (Cresson, 1902)* & $\mathrm{F}$ & NA, TL & \\
\hline Traumatomutilla spectabilis spectabilis (Gerstaecker, 1874) & $\mathrm{F}$ & Ma, PM & \\
\hline Traumatomutilla trochanterata (Gerstaecker, 1874) & $\mathrm{F}$ & TL & \\
\hline Traumatomutilla virginalis (Gerstaecker, 1874) & $\mathrm{F}$ & $\mathrm{TL}$ & \\
\hline Traumatomutilla sp. 1 & $\mathrm{~F}$ & $\mathrm{TL}$ & \\
\hline Traumatomutilla sp. 2 & $\mathrm{~F}$ & $\mathrm{TL}$ & \\
\hline Xystromutilla cornigera (Cresson, 1902)* & $\mathrm{F}$ & $\mathrm{Aq}$ & \\
\hline \multicolumn{4}{|l|}{ MUTILLINAE } \\
\hline \multicolumn{4}{|l|}{ Ephutini } \\
\hline Ephuta (Ephuta) croata (Cresson, 1902) & M & $\mathrm{C}$ & Cresson, 1902 \\
\hline Ephuta (Ephuta) gracilis (Smith, 1855) & M & $\mathrm{C}$ & Cresson, 1902 \\
\hline Ephuta (Ephuta) sp. 1 & $\mathrm{~F}$ & $\mathrm{Aq}$ & \\
\hline Ephuta (Ephuta) sp. 2 & $\mathrm{~F}$ & $\mathrm{Aq}$ & \\
\hline Ephuta (Ephuta) sp. 3 & $\mathrm{~F}$ & CG & \\
\hline Ephuta (Ephuta) sp. 4 & $\mathrm{~F}$ & CG & \\
\hline Ephuta (Ephuta) sp. 5 & $\mathrm{~F}$ & CG & \\
\hline Ephuta (Ephuta) sp. 6 & $\mathrm{~F}$ & $\mathrm{CG}$ & \\
\hline Ephuta (Ephuta) sp. 7 & $\mathrm{~F}$ & SB & \\
\hline Ephuta (Ephuta) sp. 8 & $\mathrm{~F}$ & SB & \\
\hline Ephuta (Ephuta) sp. 9 & $\mathrm{~F}$ & $\mathrm{TL}$ & \\
\hline \multicolumn{4}{|l|}{ Mutillini } \\
\hline Timulla (Timulla) corumba Mickel, 1938 & M & $\mathrm{C}$ & MicKel, 1938 \\
\hline Timulla (Timulla) daucia Mickel, 1938 & $\mathrm{~F}$ & $\mathrm{C}$ & MiCKEL, 1938 \\
\hline Timulla (Timulla) galatea Mickel, $1938^{* *}$ & $\mathrm{~F}$ & TL & \\
\hline Timulla (Timulla) intermisa Mickel, 1938 & $\mathrm{~F}$ & SB & \\
\hline Timulla (Timulla) lineoloides Mickel, 1938 & $\mathrm{~F}$ & $\mathrm{TL}$ & \\
\hline Timulla (Timulla) manni Mickel, 1938 & $\mathrm{~F}$ & SB & \\
\hline Timulla (Timulla) nasica Mickel, $1938^{* *}$ & M & $\mathrm{Ba}$ & \\
\hline Timulla (Timulla) rufiventris (Klug, 1821) & M & $\mathrm{C}$ & Cresson, 1902 \\
\hline Timulla (Timulla) scoparia (Gerstaecker, 1874) & M & Sid & MiCKel, 1938 \\
\hline Timulla (Timulla) seducta (Cresson, 1902)* & $\mathrm{F}$ & $\mathrm{TL}$ & \\
\hline Timulla (Timulla) spoliatrix Mickel, 1938 & M & $\mathrm{C}, \mathrm{I}$ & MiCKel, 1938 \\
\hline Timulla (Timulla) strigata Mickel, $1938^{* *}$ & $\mathrm{~F}$ & $\mathrm{TL}$ & \\
\hline Timulla (Timulla) terminalis (Gerstaecker, 1874)* & $\mathrm{M}, \mathrm{F}$ & BEP & \\
\hline Timulla (Timulla) tinctura Mickel, 1938 & $\mathrm{M}$ & $\mathrm{C}$ & MiCKeL, 1938 \\
\hline
\end{tabular}

conhecidas são ápteras e a maioria dos machos é alada, embora algumas espécies apresentem machos com asas reduzidas (braquípteros) ou ápteros (BROTHERs, 1975). Esse extremo dimorfismo sexual tem levado historicamente a inúmeras descrições de novas espécies, e mesmo gêneros, baseadas em um único sexo, fazendo com que a família permaneça pobremente estudada em todo o mundo (BROTHERs, 1975). Levando-se em conta a extensão geográfica de Mato Grosso do Sul, a sua grande heterogeneidade ambiental, o número reduzido de coletas ao longo da história (especialmente de machos) e o baixo número de espécies registradas para alguns gêneros que sabidamente possuem 
elevada diversidade, como Pseudomethoca, Darditilla e Ephuta, estimamos que a fauna de Mutillidae no estado deva ser superior a 130 espécies. Além disso, pelo fato de a maior parte das coletas se concentrarem na região Central do estado e na porção Sul do Pantanal-Sul, as chances de a região Norte e Leste estarem pouco representadas por espécies tipicamente encontradas no bioma do Cerrado são grandes.

Principais grupos de pesquisa e acervos. Como Mutillidae nunca contou com especialistas brasileiros, não existem pesquisadores dedicados exclusivamente ao estudo do grupo no Brasil. Até então, praticamente todos os estudos envolvendo a fauna brasileira foram ou vem sendo conduzidos por pesquisadores de outros países.

No Brasil, as principais coleções de Mutillidae encontram-se nas seguintes instituições: Coleção Entomológica Pe. Jesus Santiago Moure, Departamento de Zoologia, Universidade Federal do Paraná (DZUP), Instituto Nacional de Pesquisas da Amazônia (INPA), Museu Nacional, Universidade Federal do Rio de Janeiro (MNRJ), Museu de Zoologia da Universidade de São Paulo (MZSP) e Museu Paraense Emílio Goeldi (MPEG).

Principais lacunas de conhecimento. A ausência de sistematas trabalhando ativamente em instituições de pesquisa brasileiras é, sem dúvida, o principal motivo para a escassez de conhecimentos do grupo no país. O cenário tende a mudar com o passar dos anos, uma vez que há atualmente estudantes de pós-graduação em formação no Brasil.

Perspectivas de pesquisa para o grupo nos próximos 10 anos. As perspectivas futuras para o estudo da família no país são promissoras. Com a formação de novos sistematas (incluindo a possível criação de grupos de pesquisa nacionais) e a manutenção das colaborações com pesquisadores estrangeiros, a tendência é de que nos próximos dez anos o conhecimento e a quantidade de publicações envolvendo os mutilídeos do Brasil aumentem significantemente.

Agradecimentos. A Fundação de Apoio ao Desenvolvimento do Ensino, Ciências e Tecnologia do Estado de Mato Grosso do Sul (Fundect) e a Superintendência de Ciências e Tecnologia do Estado de Mato Grosso do Sul (Sucitec/MS) pelo convite de participação neste fascículo especial da Iheringia, Série Zoologia e o suporte financeiro para sua publicação. Aos curadores Dr. Carlos R. Brandão (MZSP), Dr. Carlos Flechtmann (MEFEIS), Dr. Gabriel A. R. Melo (DZUP) e Dr. Felipe Vivallo (MNRJ) por permitirem o acesso ao material de Mutillidae depositado em suas coleções. Ao CNPq, pelas bolsas de doutorado (143491/2011-3) e pósdoutorado (370106/2013-0) de DRL e KAW, respectivamente e a Capes, pela bolsa de doutorado de RA.

\section{REFERÊNCIAS BIBLIOGRÁFICAS}

Aranda, R. \& Catian, G. 2008. Novos registros de Mutillidae (Hymenoptera - Aculeata) para o Estado de Mato Grosso do Sul, Brasil. Revista Biociências 14(1):62-68.

Brothers, D. J. 1975. Phylogeny and classification of the aculeate Hymenoptera, with special reference to Mutillidae. The University of Kansas Science Bulletin 50(11):483-648.

BRothers, D. J. 1989. Alternative life-history styles of mutillid wasps (Insecta, Hymenoptera). In: Bruton, M. N. ed. Alternative LifeHistory Styles of Animals. Dordrecht, Kluwer Academic Publishers, p. $279-291$
BRothers, D. J. 1999. Phylogeny and evolution of wasps, ants and bees (Hymenoptera, Chrysidoidea, Vespoidea and Apoidea). Zoologica Scripta 28:233-249.

Brothers, D. J. 2006. Familia Mutilllidae. In: Hanson, P. E. \& Gauld, I. D. eds. Hymenoptera de la Región Neotropical. Memoirs of the American Entomological Institute, Issue 77. Gainesville, The American Entomological Institute. 994p.

Brothers, D. J. \& Carpenter, J. M. 1993. Phylogeny of Aculeata: Chrysidoidea and Vespoidea (Hymenoptera). Journal of Hymenoptera Research 2:227-304.

CAmbra, R. A. 1997. Comparación de la diversidad en la Sphaeropthalminae (Hymenoptera: Mutillidae) de Costa Rica y Panamá, con notas sobre biología. Scientia (Panamá) 12(2):115-128.

CAMbra, R. A. \& Quintero, D. 1992. Velvet ants of Panama: distribution and systematics (Hymenoptera: Mutillidae). In: Quintero, D. \& Aiello, A. eds. Insects of Panama and Mesoamerica. Selected Studies. Oxford, Oxford University Press, p. 459-478.

Cambra, R. A. \& Sermeño, J. M. 2001. Aportes preliminares para el conocimiento sobre la diversidad de la Mutillidae (Insecta: Hymenoptera) en El Salvador. In: MiRANDA, J. P. D. coord. Libro de Resúmenes, V Congreso de la Sociedad Mesoamericana para la Biología y la Conservación. San Salvador, p. 54.

Casal, O. H. 1968. Aportaciones para el conocimiento de las Mutillidae de la Republica Argentina. I. Las hembras del genero Darditilla (Hymenoptera). Revista de la Sociedad Entomológica Argentina 30(1-4):83-96.

Cresson, E. T. 1902. Descriptions of some Mutilla from Brazil. Transactions of the American Entomological Society 28:1-82.

Fritz, M. A. 1998. Mutillidae. In: Morrone, J. J. \& Coscarón, S. eds. Biodiversidad de Artrópodos Argentinos. Una perspectiva biotaxonómica. La Plata, Ediciones Sur, p. 445-451.

GARCIA, E. Q. 2004. Diversidade, sazonalidade, aspectos comportamentais e associação sexual de Mutillidae (Hymenoptera, Aculeata) dos Mananciais da Serra, Piraquara, Paraná, Brasil. Dissertação de Mestrado. Curitiba, Universidade Federal do Paraná. 65p.

Lelej, A. S. 2007. Biogeography of mutillid wasps (Hymenoptera, Mutillidae). In: Rasnitsyn, A.P. \& Gokhman, V. E. eds. Studies on Hymenopterous Insects. Collection of Scientific Papers. Moscow, KMK Scientific, p. 82-111.

LeLeJ, A. S. \& Brothers, D. J. 2008. The genus-group names of Mutillidae (Hymenoptera) and their type species, with a new genus, new name, new synonymies, new combinations and lectotypifications. Zootaxa 1889:1-79.

Mickel, C. E. 1938. The Neotropical mutillid wasps of the genus Timulla Ashmead (Hymenoptera: Mutillidae). Transactions of the Entomological Society of London 87:529-680.

Mickel, C. E. 1939. A monograph of the Neotropical mutillid genus Hoplomutilla Ashmead (Hymenoptera: Mutillidae). Revista de Entomologia 10(3):641-717.

Mickel, C. E. 1943. The South American genus Atillum André (Hymenoptera: Mutillidae). Revista de Entomologia 14:174-254.

Mickel, C. E. 1952. The Mutillidae (Wasps) of British Guiana. Zoologica 37(3): $105-150$

Mickel, C. E. 1960. A review of the mutillid genus Cephalomutilla André (Mutillidae: Hymenoptera). Revista Brasiliera de Entomologia 9:157168.

Mickel, C. E. 1964. A synopsis of the South American mutillid genus Reedomutilla, nom. nov. for Reedia André (nec Ashmead) (Hymenoptera). Proceedings of the Royal Society of London, Series B 33(1-2):30-36

Nonveiller, G. 1990. Catalogue of the Mutillidae, Myrmosidae and Bradynobaenidae of the Neotropical Region including Mexico (Insecta, Hymenoptera). Den Haag, SPB Academic Publishing. 150p.

Pilgrim, E. M. \& PitTs, J. P. 2006. A molecular method for associating the dimorphic sexes of velvet ants (Hymenoptera: Mutillidae). Journal of the Kansas Entomological Society 79:222-230.

Quintero, D. \& CAmbra, R. A. 1996a. Mutillidae of Paraguay. Sphecos 30:11-14.

Quintero, D. \& CAmbra, R. A. 1996b. Contribución a la sistemática de las Mutílidas (Hymenoptera) del Perú, en especial las de la Estación 
Biológica BIOLAT, Río Manu, Pakitza. In: Wilson, D. E. \& Sandoval, A. eds. Manu: The Biodiversity of Southeastern Peru. Washington, D.C., Smithsonian Institution Press, p. 327-357.

SuÁREZ, F. J. 1960. Datos sobre mutílidos neotropicales. I. Nuevas especies de Sphaerophthalminae [sic!]. EOS 36(4):451-480.
SuÁreZ, F. J. 1966. Datos sobre mutílidos neotropicales. VI. Dos nuevos géneros de Pseudomethocini de Méjico y Brasil (Hymenoptera). EOS 41(2-3):471-482.

SuÁrez, F. J. 1973. Datos sobre mutilidos neotropicales. VIII. Una nueva especie de Leucospilomutilla Ashmead (Hymenoptera). Archivos del Instituto de Aclimatacion 18:139-146. 